\title{
Interplay between morphology, structure, and electronic properties at diindenoperylene-gold interfaces
}

\author{
A. C. Dürr, ${ }^{1,2, *}$ N. Koch, ${ }^{3,4, \dagger}$ M. Kelsch, ${ }^{1}$ A. Rühm, ${ }^{1,2}$ J. Ghijsen, ${ }^{5}$ R. L. Johnson, ${ }^{6}$ J.-J. Pireaux, ${ }^{5}$ J. Schwartz,${ }^{4}$ \\ F. Schreiber, ${ }^{1,2, \sharp}$ H. Dosch, ${ }^{1,2}$ and A. Kahn ${ }^{3}$ \\ ${ }^{1}$ Max-Planck-Institut für Metallforschung, Heisenbergstraße 3, 70569 Stuttgart, Germany \\ ${ }^{2}$ Universität Stuttgart, Pfaffenwaldring 57, 70550 Stuttgart, Germany \\ ${ }^{3}$ Department of Electrical Engineering, Princeton University, Princeton, New Jersey 08544, USA \\ ${ }^{4}$ Department of Chemistry, Princeton University, Princeton, New Jersey 08544, USA \\ ${ }^{5}$ Laboratoire Interdisciplinaire de Spectroscopie Electronique, Facultés Universitaires Notre-Dame de la Paix, B-5000 Namur, Belgium \\ ${ }^{6}$ II. Institut für Experimentalphysik, Universität Hamburg, D-22761 Hamburg, Germany \\ (Received 1 April 2003; revised manuscript received 12 June 2003; published 29 September 2003)
}

\begin{abstract}
We present a study of the morphology, structure, and electronic properties of interfaces formed between $\mathrm{Au}$ and the organic semiconductor diindenoperylene (DIP) employing transmission electron microscopy (TEM), atomic-force microscopy (AFM), x-ray diffraction, and ultraviolet photoelectron spectroscopy (UPS). Pronounced islanding of the DIP films deposited on Au is found by AFM as well as by TEM. In addition, TEM images show individual monolayers of DIP with the long molecular axis parallel to the substrate, suggesting a lying-down phase ( $\lambda$-phase). TEM images also show the formation of Au clusters and a certain degree of Au interdiffusion into the DIP film after Au deposition on DIP. Specular and grazing incidence x-ray diffraction show the coexistence of standing phase ( $\sigma$-phase) and $\lambda$-phase with a preferred growth of the $\lambda$-phase. UPS is used to study the evolution of the electronic structure of the DIP-on-Au and Au-on-DIP interfaces. DIP is found to physisorb on Au. The energy difference between substrate Fermi level and the DIP highest occupied molecular orbital at the interface is $1.0 \mathrm{eV}$. This hole-injection barrier increases to $1.45 \mathrm{eV}$ away from the interface because of decreased screening by the metal and possible changes in molecular conformation. For Au deposition onto DIP, UPS traces the formation of Au clusters as a function of Au coverage. These clusters percolate only for Au coverages higher than $32 \AA$ to give a continuous metal surface coverage and conductivity. The interaction between the Au clusters and DIP is also found to be of physisorptive nature.
\end{abstract}

DOI: 10.1103/PhysRevB.68.115428

PACS number(s): 68.55.-a, 68.37.- d, 68.43.- h, 73.61.- r

\section{INTRODUCTION}

A steady increase in the number of reports on new electronic and optoelectronic devices based on conjugated organic materials can be noticed over the past few years. ${ }^{1-6}$ Key elements of device performance are the structure of the functional organic layer and the charge injection from metal contacts. Therefore, a thorough understanding of organic film structure and morphological and electronic properties of metal/organic interfaces is essential for applications in future devices. Device function and efficiency are frequently evaluated assuming abrupt, homogeneous interface morphology, and interface energetics based on separately determined material parameters (work function, ionization energy, and electron affinity). However, several reports have made clear that these assumptions are often not valid. Diffusion, chemical reactions, and polarization of different surrounding matter, can, if occurring at the interface, lead to properties significantly different from the expected ones. ${ }^{7-13}$

It was recently demonstrated that thin films of diindenoperylene [DIP, $\mathrm{C}_{32} \mathrm{H}_{16}$, see Fig. 1(a)] deposited on $\mathrm{SiO}_{2}$ form smooth closed layers with high structural order in which the molecules (almost exclusively) stand upright with their long axis aligned along the surface normal $\left[d_{\text {DIP }}(001)\right.$ $\approx 16.6 \AA] .{ }^{14}$ This standing phase is denoted by $\sigma$-phase in what follows, see the left-hand side of Fig. 1(b). It was also shown that these films exhibit a high hole mobility of up to
$0.1 \mathrm{~cm}^{2} / \mathrm{Vs}$ in the plane parallel to the overlapping $\pi$-orbitals in field-effect transistor geometry, i.e., parallel to the substrate surface. ${ }^{15,16}$ This makes DIP interesting for application in thin-film transistors or as hole-transport layer in other organic devices, e.g., organic light emitting diodes (OLED's). High-work-function metals such as Au are then presumed to be adequate for hole injection with low barrier. The morphology of interfaces between Au and DIP deposited on $\mathrm{SiO}_{2}$ have already been investigated. ${ }^{13,17}$

When DIP is deposited on $\mathrm{Au}$ rather than on $\mathrm{SiO}_{2}$, the molecule-substrate interaction changes significantly and may result in a different film morphology, including the formation of a lying-down phase (denoted by $\lambda$-phase in what follows) where the long axis of the molecule is parallel to the substrate [right-hand side of Fig. 1(b)], as already observed for a variety of other molecule-on-metal systems. ${ }^{18-22}$

In the present study we conducted a detailed investigation of structure and (interfacial) morphology of DIP/Au and Au/ $\mathrm{DIP} / \mathrm{Au}$, as well as of the electronic structure and energylevel alignment at DIP-on-Au and Au-on-DIP interfaces prepared under ultrahigh vacuum (UHV) conditions. We employed atomic-force microscopy (AFM) to investigate the surface morphology of DIP/Au samples in real space, crosssectional transmission electron microscopy (TEM) to determine the interfacial morphology between Au and DIP, and specular and grazing incidence $\mathrm{X}$-ray scattering ${ }^{23-25}$ to determine the structure of the thin-film system in out-of-plane 


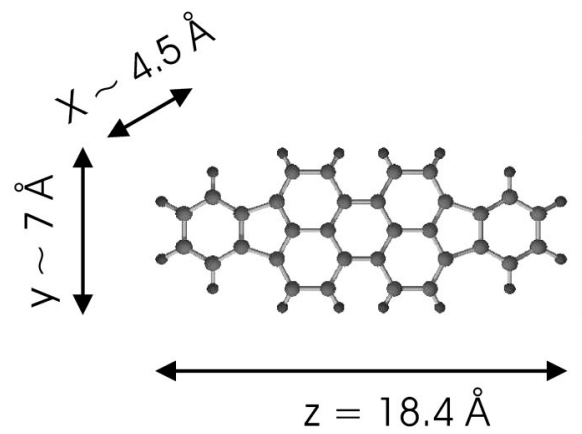

(a)

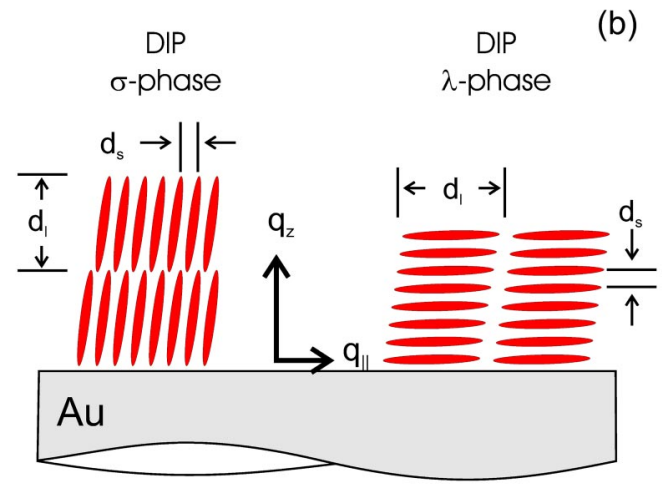

FIG. 1. (a) Structure of the DIP molecule. (b) Sketch of the different growth modes of DIP molecules. Left: $\sigma$-phase, the molecules are stacked with their long axis essentially perpendicular to the substrate. Right: $\lambda$-phase, the molecules are lying flat on the surface. The lattice spacing $d_{l}$ is uniquely related to the long $(z)$ axis of the molecule, whereas several lattice constants $d_{s}$ may be probed, depending on the crystalline arrangement of the DIP molecules around the long molecular axis.

(perpendicular to the surface) and in-plane (parallel to the surface) directions, respectively. Ultraviolet photoemission spectroscopy (UPS) has been used to investigate the electronic properties of the DIP/Au and the Au/DIP interface.

\section{EXPERIMENT}

The samples were prepared on oxidized (4000 $\AA$ ) silicon wafers with a (100) orientation precoated with a $1000 \AA$ thick polycrystalline Au layer on a $100 \AA \mathrm{Cr}$-adhesion layer: $\mathrm{Au} / \mathrm{Cr} / \mathrm{SiO}_{2} / \mathrm{Si}(100){ }^{26}$ After insertion in UHV (base pressure below ca. $5 \times 10^{-10}$ mbar) the substrates were cleaned by Ar-ion sputtering, and a fresh Au layer (ca. $300 \AA$ A) was deposited to provide a clean metal surface. ${ }^{27}$ Approximately $300 \AA$ of DIP (purchased from Institut für PAH-Forschung, Greifenberg, Germany) was deposited onto the substrates (ca. $300 \AA$ ). On some samples, ca. $150 \AA$ of Au was deposited onto the DIP/Au heterostructure. Both DIP and Au were deposited at room temperature and at a rate of ca. $2 \AA / \mathrm{min}$. The mass thickness of the DIP (bulk density-1.35 g/ $\mathrm{cm}^{3}$ ) and $\mathrm{Au}$ (bulk density-19.3 $\mathrm{g} / \mathrm{cm}^{3}$ ) layers was monitored with a quartz-crystal microbalance (QCM). No corrections for possible differences in sticking coefficient between QCM and actual samples were made. For the UPS study, the materials were deposited incrementally, and after each deposi-
TABLE I. Description of the analyzed samples. $\Theta_{\mathrm{DIP}}$ and $\Theta_{\mathrm{Au}}$ denote the nominal coverage of DIP and $\mathrm{Au}$, on the $\mathrm{Au} / \mathrm{Cr} / \mathrm{SiO}_{2} / \mathrm{Si}(100)$ substrates, respectively.

\begin{tabular}{lccc}
\hline \hline Sample no. & $\begin{array}{c}\Theta_{\mathrm{DIP}} \\
(\AA)\end{array}$ & $\begin{array}{c}\Theta_{\mathrm{Au}} \\
(\AA)\end{array}$ & Analyzed by \\
\hline 1 & 300 & 0 & AFM/x-ray \\
2 & 300 & 150 & TEM \\
3 & 0 & 0 & specular $\mathrm{x}-\mathrm{ray}$ \\
4 & 300 & 130 & UPS/(TEM) \\
\hline \hline
\end{tabular}

tion step the samples were transferred into the analysis chamber (without breaking UHV) to record UPS spectra. For details of individual sample properties see Table I.

AFM measurements were carried out in tapping mode with a Digital Instrument Nanoscope III at ambient conditions. To avoid edge effects in the determination of the rootmean-square (rms) surface roughness $\sigma_{r m s}$, increasingly large scan ranges were applied until $\sigma_{r m s}$ became independent of the range.

TEM images were taken with a Philips CM 200 microscope operated at $200 \mathrm{kV}$. The preparation of specimens for cross-sectional TEM imaging is described elsewhere. ${ }^{28}$

$\mathrm{X}$-ray-diffraction measurements were performed at the MPI beamline at the Angstrom-Quelle Karlsruhe (ANKA). ${ }^{29}$ A horizontal sample geometry and an energy of $E=10 \mathrm{keV}$ corresponding to $\lambda=1.24 \AA$ were used for all measurements. Figure 2 shows a schematic view of the beamline setup, sketching the slit locations and the employed scattering geometries.

The angular resolution for the specular scans as defined by the slit settings was $\Delta 2 \Theta=0.035^{\circ}$ for scans at angles $2 \Theta$ $>10^{\circ}$ and $\Delta 2 \Theta=0.014^{\circ}$ for $2 \Theta<10^{\circ}$.

To probe lattice constants parallel to the surface [see Fig. 1(b)], detector scans were carried out in grazing-incident $\mathrm{x}$-ray-diffraction (GIXD) geometry. The GIXD measurements were performed at an incident angle $\alpha_{i}=0.15^{\circ}$ corresponding to the critical angle $\alpha_{c}$ for total external reflection of the DIP film. Since this angle is below the critical angle of total external reflection of the Au film $\left(\alpha_{c, A u} \approx 0.7^{\circ}\right)$, the $\mathrm{x}$-ray wave only penetrates several angstroms into the substrate, and therefore this surface sensitive GIXD measurement probes mainly the in-plane structure $\left(q_{\|}\right)$of the DIP film, see Fig. 1(b). ${ }^{23,24}$ Furthermore, a measurement with $\alpha_{i}=0.7^{\circ} \approx \alpha_{c, A u}$ was carried out, which allows for the penetration of x-rays into the substrate and considerably enhances the contribution of scattering intensity from the substrate. For the GIXD measurements we set $\sigma_{i H}=1.0 \mathrm{~mm}$ and $\sigma_{i V}=0.5 \mathrm{~mm}$. Setting the vertical detector slit $\sigma_{f V}$ $=15.0 \mathrm{~mm}$ in combination with $\alpha_{f}=0.375^{\circ}$ for $\alpha_{i}=0.15^{\circ}$ ( $\alpha_{f}=0.375^{\circ}$ for $\alpha_{i}=0.7^{\circ}$ ) allows for an integration of the scattered intensity along the surface normal with an acceptance angle of $\approx 0.9^{\circ} . \sigma_{f H}$ was set to $1.0 \mathrm{~mm}$ in survey scans, and to $0.3 \mathrm{~mm}$ to determine the individual peak widths with enhanced resolution.

To analyze the diffraction-peak positions and peak widths, the intensity was plotted as a function of $q_{z}$ (perpendicular to the surface) and $\left|q_{\|}\right|$(parallel to the surface) in specular and 

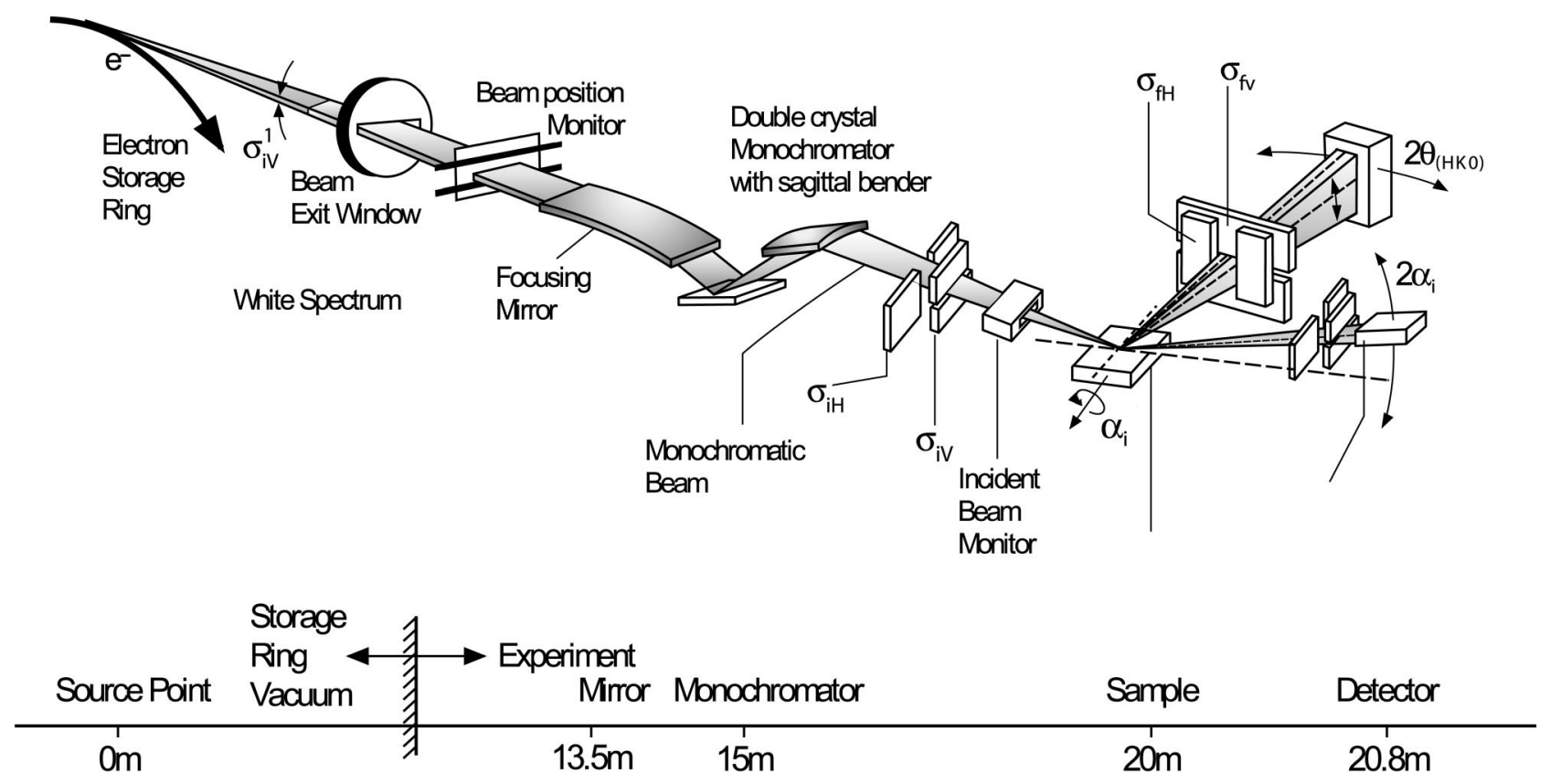

FIG. 2. Schematic view of the experimental setup for specular and grazing-incidence x-ray diffraction. The beam optics elements are taken from the MPI beamline at ANKA. $\sigma_{i V}$ and $\sigma_{i H}$ are the vertical and horizontal slits in front of the sample, whereas $\sigma_{f V}$ and $\sigma_{f H}$ are the detector slits governing the resolution of the experiment. $\alpha_{i}$ denotes the incident angle of the x rays on the sample. $2 \Theta$ is the detector angle in grazing-incident $\mathrm{x}$-ray-diffraction scans. In specular scans, $2 \Theta_{(H K 0)}$ is set to $0^{\circ}$ and the detector angle is set to $2 \alpha_{i}$.

GIXD mode, respectively. Subsequently the peaks were fitted with Gaussians giving the peak center $q^{*}$ and the full width at half maximum of the peak, $\Delta q$. The corresponding lattice constant is determined as $d=2 \pi / q^{*}$, whereas a lower limit for the minimal domain size is given by $D^{*}$ $=2 \pi / \Delta q$.

Photoemission experiments were performed at the FLIPPER II beamline in HASYLAB at DESY. ${ }^{30}$ The resolution of the electron spectrometer was $\approx 0.15 \mathrm{eV}$ (width of the intensity drop from $80 \%$ to $20 \%$ on the Au Fermi edge). In order to record the secondary electron cutoff and determine the position of the vacuum level, the samples were negatively biased with respect to the electron spectrometer $(-16 \mathrm{~V}) .^{31}$ The energy positions of the cutoff and the low bindingenergy onset of the highest occupied molecular orbital (HOMO) were determined by linear extrapolation to the background. The work function is determined as the difference between the incident photon energy and the total width of the energy distribution curve. We estimate the error in the position determination to be smaller than $\pm 0.1 \mathrm{eV}$. The photon energy was set to $22 \mathrm{eV}$. UPS measurements were performed at room temperature. To avoid charging of the sample surface after Au deposition, the sample was illuminated by the light of a standard halogen lamp during the UPS measurements. ${ }^{32}$ The UPS spectra were fitted using "WINSPEC," a program developed at the University of Namur, Belgium.

For the assignment of the HOMO and HOMO-1 of the DIP films, density-functional-theory calculations on a single DIP molecule was done using the B3LYP functional with the 6-31G(d) basis set, as implemented in the program package
GAUSSIAN $98^{33}$ for geometry optimization and calculation of electron energy eigenvalues.

The eigenvalues were broadened with a Gaussian function (width $=0.3 \mathrm{eV}$ ) to simulate a UPS spectrum, a common procedure to compare the theoretically obtained eigenenergies to the photoemission spectra. ${ }^{34,35}$

The calculated spectrum was then shifted to align with the experimentally obtained UPS spectrum of the $300 \AA$ thick DIP film.

\section{RESULTS AND DISCUSSION}

\section{A. Morphology of DIP on Au (AFM)}

Figure 3(a) displays the morphology of sample no. 1, consisting of nominally $300 \AA$ of DIP deposited on the Au substrate at HASYLAB (see Table I). The surface shows pronounced islanding and exhibits a roughness of $\sigma=215 \pm 15$ $\AA$. The maximum corrugation is of the order of $1500 \AA$. For comparison, Fig. 3(b) shows the morphology of $400 \AA$ DIP/ $\mathrm{SiO}_{2}$, as measured in two previous studies by AFM, using approximately the same lateral scan-range. ${ }^{14,36}$ The surface shows a terrace morphology, which reflects the presence of layers of essentially upright standing molecules grown in the $\sigma$-phase with lattice constant $d \approx 16.6 \AA$. The surface exhibits a roughness of $\sigma_{r m s}=28 \pm 3 \AA$, and a maximum corrugation of the order of $120 \AA$.

These very different growth modes can be attributed to different substrate-molecule interactions, since the surface morphology of the Au substrate alone $\left(\sigma_{r m s} \leqslant 10 \AA\right)$ cannot account for the pronounced islanding. In addition, the absence of well-defined terraces in sample no. 1 (even at 

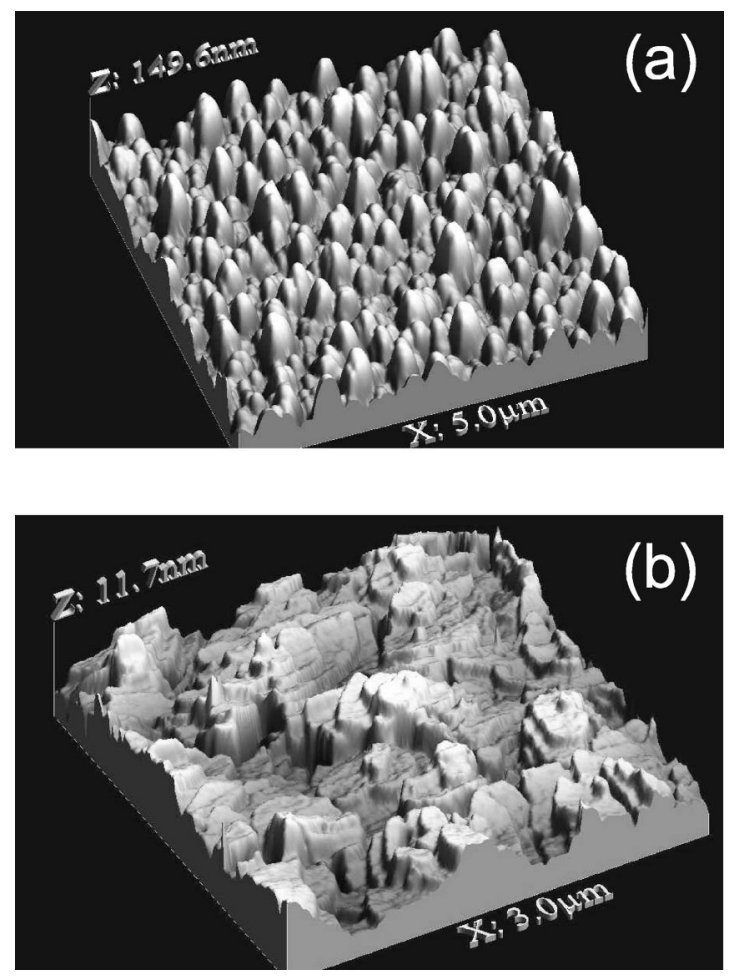

FIG. 3. (a) Three-dimensional (3D) representation of an AFM image of sample no. 1. (b) 3D representation of an AFM image of a $\operatorname{DIP}(400 \AA) / \mathrm{SiO}_{2}$ sample.

smaller scan ranges) already indicates that DIP does not necessarily exhibit $\sigma$-phase growth if deposited on $\mathrm{Au}$.

\section{B. Interfacial morphology of Au/DIP/Au and structure of DIP/Au (TEM)}

Figure 4(a) shows a cross-sectional TEM image through the $x-z$ plane of the Au/DIP/Au heterostructure of sample no. $2(150 \AA \mathrm{Au} / 300 \AA \mathrm{DIP}$, see Table I) with a lateral range of ca. $1 \mu \mathrm{m}$. The image clearly shows the islandlike morphology of the DIP film, with island heights of up to $\approx 1500 \AA$, consistent with the AFM results discussed above. The Au layer deposited on the DIP film covers the islands with a relatively sharp interface and consists of small, coalesced clusters rather than of a smooth, closed film. In addition, interdiffusion of smaller Au clusters into the DIP islands is seen. However, the pronounced islanding morphology of DIP makes it difficult to identify which Au clusters are embedded into individual DIP islands. Because of the relatively large probed sample thickness of $\approx 100 \mathrm{~nm}$ (along the $y$ direction in Fig. 4), Au clusters simply covering the top of a small DIP island located in front of a taller DIP island may appear as clusters interdiffused into the taller DIP island.

Figure 4(b) displays a close-up of a single DIP island. One can see a stripe pattern extending over the whole lateral length of the DIP island, which is almost normal to the Ausurface (tilt-angle $\phi=17^{\circ} \pm 5^{\circ}$ with respect to the surface normal). Each pair of dark-bright contrast can be attributed to a single-crystalline plane standing approximately perpendicular to the surface. The observation of this stripe pattern is evidence for high crystalline order in the DIP-islands. Closer inspection of the stripe pattern reveals a separation between individual crystalline planes of $d=16 \pm 2 \AA$ [ca. $11-14$ crystalline planes per $200 \AA$, see inset of Fig. 4(b)]. This distance is attributed to the stacking of DIP along the long axis of the molecule $\left[d_{l}\right.$ in Fig. $\left.1(\mathrm{~b})\right]$, forming a highly ordered $\lambda$-phase. The same observations were made with cross-sectional TEM on specimens made from sample no. 4.

A more detailed insight into the crystalline order and the lattice constants is provided by the $\mathrm{x}$-ray-diffraction measurements discussed in the following section.

\section{Structure of DIP / Au}

\section{Crystallographic aspects}

For the analysis of the results from specular and grazingincidence $\mathrm{x}$-ray diffraction, it is useful to make some introductory remarks about the possible crystallographic structures of the DIP film and about the characteristics of the corresponding X-ray-diffraction patterns.

A possible arrangement of DIP molecules in a DIP unit cell (UC) - for simplicity in a two-dimensional (2D) projection along the long molecule axis-is shown in Fig. 5(a). The structure in Fig. 5(a) is called herring-bone-structure (HBS) and is often observed in organic crystals. ${ }^{37}$ The $2 \mathrm{D}$ UC is characterized by two lattice parameters $a$ and $b$, enclosing an angle $\gamma$ close to $90^{\circ}$. The corresponding unit cell in reciprocal space is depicted in Fig. 5(b). Its lattice parameters are given by $\bar{a}=2 \pi /(a \sin \gamma)$ and $\bar{b}=2 \pi /(b \sin \gamma)$, and the enclosed angle is $\bar{\gamma}=\pi-\gamma$. As can be seen from the figure, mixed reflections ( $h k$ ) with $h k \neq 0$ are split in two, e.g., (11) and $(\overline{1} 1)$ for $\gamma \neq 90^{\circ}$. Depending on the number and the arrangement of the DIP molecules inside the UC, certain Bragg reflections may be forbidden. In the case of the HBS sketched in Fig. 5(a) with two molecules per UC and for the special case $\gamma=90^{\circ}$, the UC belongs to the space group $p 2 g g$ for which the following selection rules apply: $(h 0)$ and $(0 k)$ reflections are forbidden for odd $h$ and $k$, respectively; there are no conditions for other $(h k)$ reflections.

In earlier GIXD experiments on DIP films grown in the $\sigma$-phase on $\mathrm{SiO}_{2}$ and $\mathrm{Al}_{2} \mathrm{O}_{3}$ three reflections at $q_{\|}$ $=1.15 \AA^{-1}, q_{\|}=1.47 \AA^{-1}$, and $q_{\|}=1.72 \AA^{-1}$ have been observed. ${ }^{38}$ These can be assigned to a UC characterized by the following parameters: $a=8.55 \AA, b=7.09 \AA$, and $\gamma=90^{\circ}$. The three reflections correspond to the $(11)_{\sigma}$, $(20)_{\sigma}$, and $(21)_{\sigma}$ reflections. The subscript $\sigma$ indicates that the reflections belong to the $\sigma$-phase and are therefore inplane reflections. The absence of a (10) and a (01) reflection is evidence for the presence of two molecules in the UC, corresponding to the selection rules discussed above. The aspect ratio $b / a$ is $1: 1.206$. Given that two molecules are present, the occupied area per molecule is $30.31 \AA^{2}$ parallel to the substrate, which is of the order of the area spanned by the two short axes of the DIP molecule.

\section{Specular $x$-ray scattering (structure along the surface normal)}

Figure 6(a) displays specular scans along the surface normal recorded on the $\mathrm{Au}$ substrate (sample no. 3) and on 

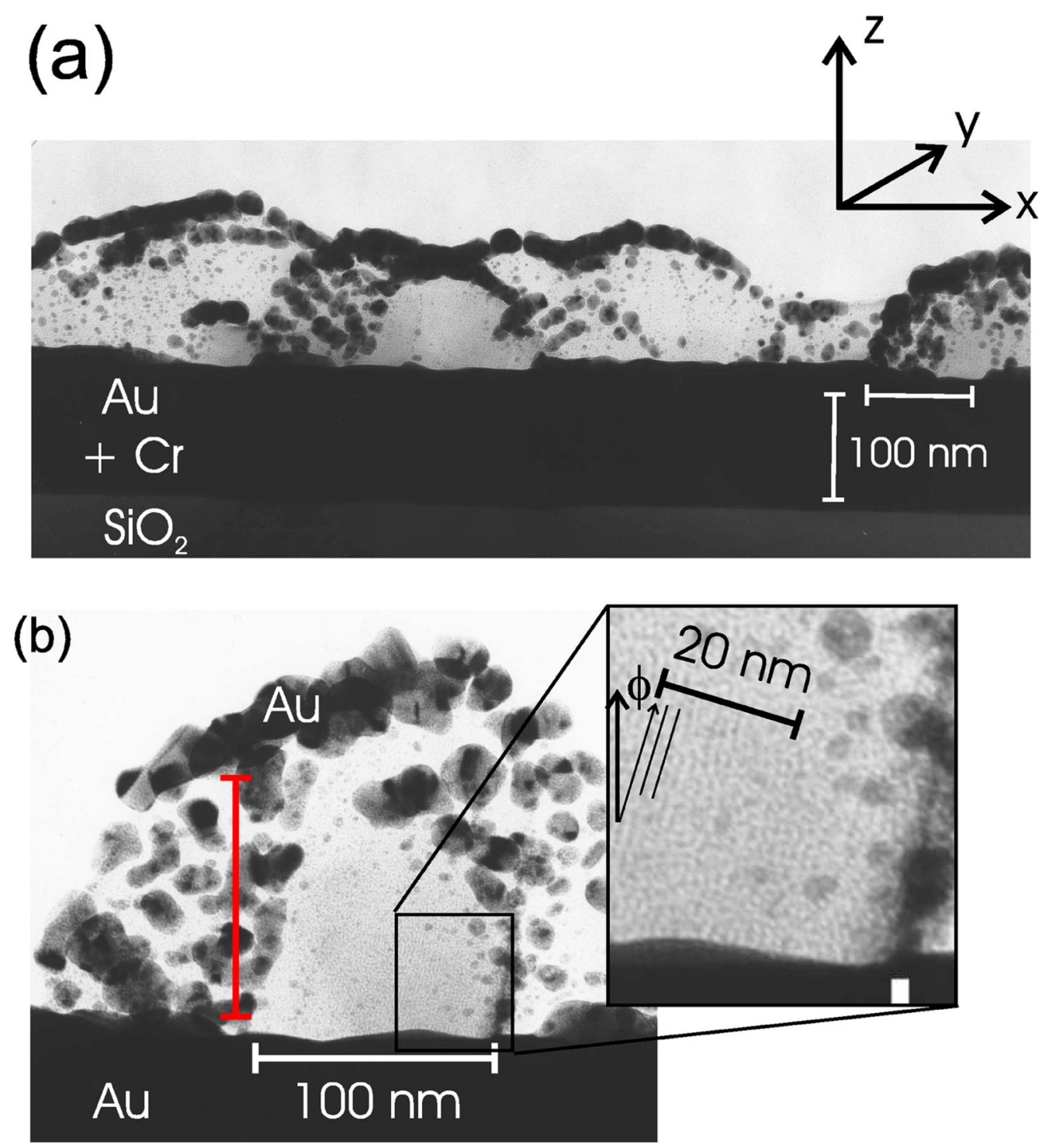

FIG. 4. (a) Cross-sectional TEM image of sample no. 2. (b) Close-up into a cross-sectional TEM image of a distinct DIP island covered with Au clusters. Individual DIP crystalline planes are seen as nearly perpendicular pairs of dark-bright contrast. In the magnified view, three of the crystalline planes are highlighted by lines to clarify the orientational angle $\phi$ of the planes with respect to the substrate and to determine the lattice constant of these planes, which is found to be $d_{\mathrm{DIP}}=16 \pm 2 \AA$.

sample no. 1. The data for both samples exhibit two dominant Bragg reflections at $q_{z}=2.67 \AA^{-1}$ and $q_{z}=3.08 \AA^{-1}$. These are attributed to Au grains of the substrate with (111) and (200) orientation along the surface normal. The coexistence of both reflections in the specular scan is evidence of polycrystallinity of the Au substrate. A comparison of the two scans clearly demonstrates that the additional Bragg reflections of sample no. 1 below $q_{z}=2.67 \AA^{-1}$ arise from the DIP layer.

Figure 6 (b) shows a close-up of the specular scan of sample no. 1 covering the region $0.95 \AA^{-1}<q_{z}$ $<1.95 \AA^{-1}$. Six Bragg reflections can clearly be discerned, five of which are associated with lattice plane spacings $d_{D I P}$ below $5.5 \AA$ A. Since the present scan probes lattice plane spacings along the surface normal (out of plane) this observation is evidence of the presence of DIP grown in a $\lambda$-phase [see Fig. 1(b)]. The three reflections marked with arrows in Fig. 6(b) are at the same positions as the in-plane reflections of the $\sigma$-phase discussed above. Therefore, it is consistent with the position of these reflections that the $\lambda$-phase exhibits the same HBS structure and thus the same UC as the $\sigma$-phase, yet with the long molecular axis rotated into the surface plane. The reflection at $q_{\|}=1.77 \AA^{-1}$ can also be explained with this $\mathrm{UC}$ as a $(02)_{\lambda}$ reflection. To explain the coexistence of four different $\lambda$ reflections, however, we have to assume a fiberlike structure of the $\lambda$-phase, i.e., the DIP material is textured with the long molecular axis parallel to the surface and the crystallites are rotated more or less randomly around that axis.

An analysis of the peak widths leads to an estimate of the domain size of the $\lambda$-phase of the order of $D_{\lambda}^{* \perp}$ $=300 \AA-400 \AA$ along the surface normal. 
(a)
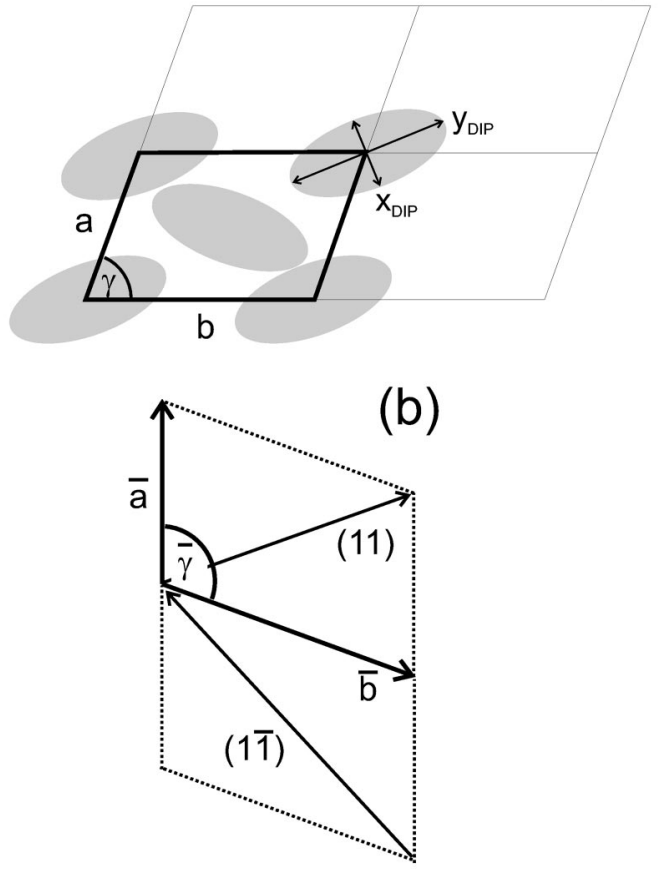

FIG. 5. (a) Schematic representation of a possible UC of the DIP $\sigma$-phase in the $x-y$ plane [as defined in Fig. 1(a)]. $a$ and $b$ are the UC axes and $\gamma$ is the angle between the axes. (b) Reciprocal unit cell with $\bar{\gamma}=\pi-\gamma$. It can be seen that the (11) and the (1) lattice vectors have different lengths.

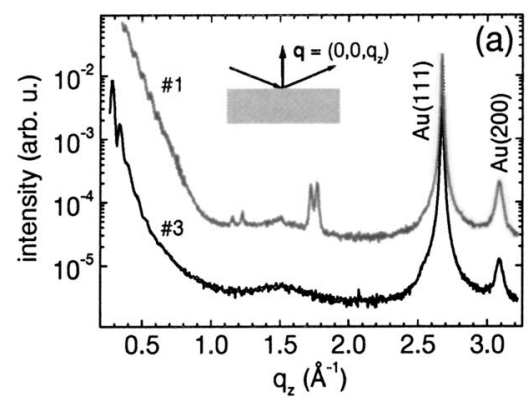

Figure 6(c) shows a close-up of the specular scan of sample no. 1 covering the region $0.3 \AA^{-1}<q_{z}<0.8 \AA^{-1}$. The data exhibit intensity oscillations (so-called Kiessig fringes) with two different periodicities which are due to interlayer x-ray interference effects. ${ }^{25}$ The periodicities can be associated with the thickness of the Cr-adhesion layer $\left(D_{C r} \approx 110 \AA\right)$ and the Au layer $\left(D_{A u} \approx 800 \AA\right)$ through the relation $D=2 \pi / \Delta Q$, where $\Delta Q$ denotes the periodicity of the individual oscillations as indicated in the figure. The almost undamped oscillations allow one to estimate the surface roughness of the Au layer to be less than $10 \AA$ (as mentioned before). Superimposed on the intensity oscillations are two rather weak Bragg reflections at $q_{z} \approx 0.38 \AA^{-1}$ and at $q_{z}$ $\approx 0.76 \AA^{-1}$ (indicated by arrows) corresponding to the firstand second-order reflection of DIP lattice planes with a spacing of $d \approx 16.6 \AA$ perpendicular to the substrate. This is evidence of some DIP molecules grown in the $\sigma$-phase, i.e., for a stacking of molecules along the surface normal with an interlayer spacing that is determined by the length of the long molecule axis $\left[d_{l}\right.$ in Fig. 1(b)]. Note that the weak reflection at $q_{z}=1.51 \AA^{-1}$ in Fig. 6(b) can also be assigned to the $\sigma$-phase as a higher-order reflection [(004) reflection]. As for the $\lambda$-phase, we can assume that the $\sigma$-phase crystallites are rotated randomly around the long axis (fiberlike structure) due to the polycrystalline nature of the Au substrate.

The $q z$ positions of the discussed reflections and the derived lattice spacings $d_{\perp}$ along the surface normal are summarized in Table II. The assignment of the reflections to the various crystalline orientations is also indicated in the table.

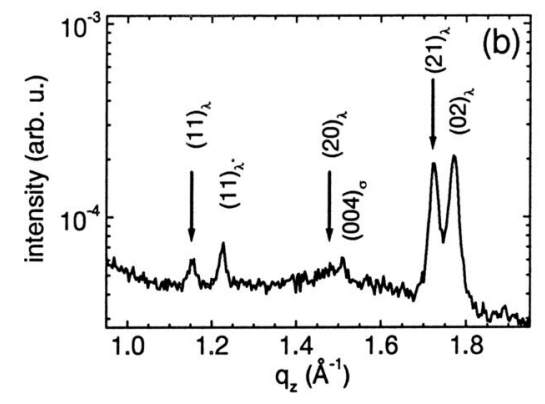

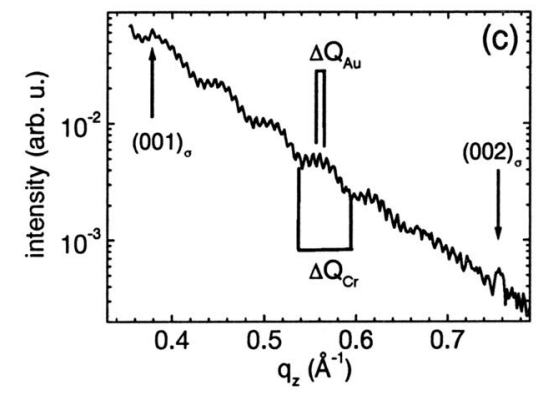

FIG. 6. (a) Specular x-ray diffraction scans from sample nos. 1 and 3 (lower curve). For clarity, the curves are shifted with respect to each other. The inset schematically shows the scattering geometry for the specular scans. (b) Magnified view of the specular x-ray-diffraction data (region $0.95 \AA^{-1}<q_{z}<1.95 \AA^{-1}$ ) for sample no. 1 (upper curve). The arrows mark the positions in $q_{z}$ where in-plane Bragg reflections were found on DIP samples grown in the $\sigma$-phase $\left[\mathrm{DIP} / \mathrm{SiO}_{2}\right.$ and $\mathrm{DIP} / \mathrm{Al}_{2} \mathrm{O}_{3}$, (Ref. 38)]. (c) Magnified view of the specular x-raydiffraction data (region $0.3 \AA^{-1}<q_{z}<0.8 \AA^{-1}$ ) for sample no. 1. The arrows mark the position of weak Bragg reflections which correspond to the $(001)_{\sigma}$ and $(002)_{\sigma}$ DIP reflections of $\sigma$-phase DIP (Refs. 14 and 38). The periodicity of the intensity oscillations, $\Delta Q$, is related to the thickness of the $\mathrm{Au}$ and the Cr layer, respectively. 
TABLE II. Position of the specular Bragg reflections of the DIP/Au sample (no. 1) and of the substrate (no. 3). $q_{z}^{*}$ values in bold letters have already been observed as in-plane reflections of DIP grown in $\sigma$-phase in previous studies. The calculations have been carried out assuming the following UC parameters: $a$ $=8.55 \AA, b=7.09 \AA, \gamma=90^{\circ}$. Subscripts $\sigma$ and $\lambda$ indicate to which phase the labeled reflections are assigned.

\begin{tabular}{ccccc}
\hline \hline Sample no. & $\begin{array}{c}q_{z}^{*} \\
\left(\AA^{-1}\right)\end{array}$ & $\begin{array}{c}d_{\perp} \\
(\AA)\end{array}$ & $\begin{array}{c}\text { Assigned } \\
\text { to }\end{array}$ & $\begin{array}{c}\text { Calculation } \\
\left(\AA^{-1}\right)\end{array}$ \\
\hline \multirow{2}{*}{1} & $0.37(8)$ & $16.61 \pm 0.10$ & $(001)_{\sigma}$ & \\
& $0.75(8)$ & $16.58 \pm 0.05$ & $(002)_{\sigma}$ & \\
& $\mathbf{1 . 1 5 ( 4 )}$ & $5.45 \pm 0.02$ & $(11)_{\lambda}$ & 1.1513 \\
& $\mathbf{1 . 4 8 ( 3 )}$ & $4.24 \pm 0.03$ & $(20)_{\lambda}$ & 1.4698 \\
& $1.51(0)$ & $16.64 \pm 0.03$ & $(004)_{\sigma}$ & \\
& $\mathbf{1 . 7 2 ( 5 )}$ & $3.64 \pm 0.02$ & $(21)_{\lambda}$ & 1.7163 \\
& $1.77(0)$ & $3.55 \pm 0.02$ & $(02)_{\lambda}$ & 1.7724 \\
& $2.67(4)$ & 2.35 & $\mathrm{Au}(111)$ & \\
& $3.08(5)$ & 2.04 & $\mathrm{Au}(200)$ & \\
3 & $2.673(3)$ & 2.35 & $\mathrm{Au}(111)$ & \\
& $3.084(9)$ & 2.04 & $\mathrm{Au}(200)$ & \\
\hline \hline
\end{tabular}

The combination of all the information obtained from the specular scans provides evidence for the coexistence of $\lambda$-phase(s) [Fig. 6(b)] and $\sigma$-phase [Fig. 6(c)].

We note, however, that there is, to our knowledge, no example of $\sigma$-phase growth of similar organic molecules on (noble) metal substrates in the literature and, thus, the existence of $\sigma$-phase growth on a bare noble metal substrate is very unlikely. A qualitative discussion of the underlying physical processes of this issue is given below. Based on these considerations we assume that the first monolayer forms a $\lambda$-phase only, and that the growth of $\sigma$-phase domains is mediated by defects or grain boundaries of the underlying layer(s) of $\lambda$-phase DIP. The polycrystalline Au substrate may govern this evolution due to its high density of grain boundaries reproduced by the first DIP monolayers rather than due to its various crystallographic orientations.

\section{GIXD (in-plane structure)}

Figure 7 shows the intensity distribution as a function of $\left|q_{\|}\right|$(i.e., a "powder spectrum") as obtained by detector scans $(2 \Theta)$ in GIXD scattering geometry.

As indicated in the figure, the two scans were recorded at two different incident angles $\alpha_{i}$. The scans reveal several Bragg reflections. For technical reasons, it was not possible to carry out azimuthal scans at the position of individual Bragg reflections, yet no preferential azimuthal orientation is expected due to the polycrystalline nature of the $\mathrm{Au}$ substrate.

An analysis of the peak width of the $\mathrm{Au}(111)$ and $\mathrm{Au}(200)$ reflection gives a lower limit of the associated lateral domain sizes of $\approx 250 \AA$ and $\approx 130 \AA$, respectively.

The strongest Bragg reflection for $\alpha_{i}=0.15^{\circ}$ at $\left|q_{\|}\right|$ $=0.376 \AA^{-1}$ corresponds to a lattice constant $d_{l}=16.7 \AA$. This is evidence that a large fraction of the DIP grows in the $\lambda$-phase. The reflection can be assigned to the $(001)_{\lambda}$ reflec-

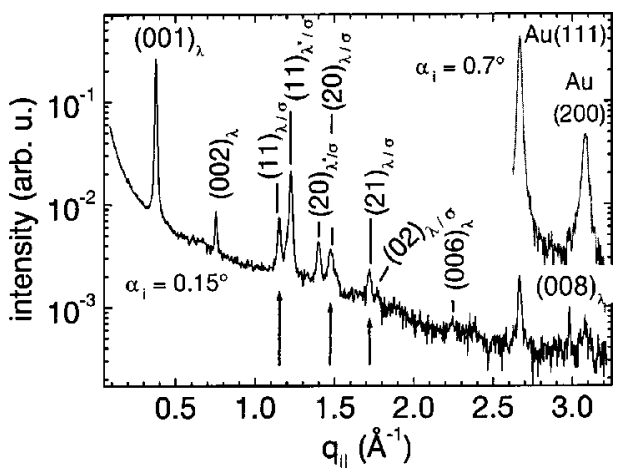

FIG. 7. GIXD measurements on sample no. 1 under two different angles of incidence. Arrows mark the peak positions at which in-plane reflections have already been found in previous studies of DIP grown (almost exclusively) in the $\sigma$-phase (Ref. 38).

tion of DIP [the index (001) for stacking along $d_{l}$ was chosen to allow for a comparison with previous studies $\left.^{13,14,28}\right]$. The occurrence of the $(002)_{\lambda}$ Bragg reflection at $\left|q_{\|}\right|=0.754 \AA^{-1}$ shows the high crystalline order of $\lambda$-phase domains. An analysis of the peak widths of both reflections provides an estimate for the associated domain size of $D_{\lambda}^{* \|} \geqslant 600 \AA$ parallel to the surface, which is consistent with the results of the TEM analysis [Fig. 4(b)] and larger than the Au-grain size. A comparison with the specular measurements discussed in the preceding section shows that the $d_{l}$ lattice spacing in the $\lambda$-phase(s) $(16.7 \AA$ parallel to the surface) is almost identical to the corresponding lattice spacing in the $\sigma$-phase (16.6 $\AA$ perpendicular to the surface).

Nearly all other Bragg reflections (see Fig. 7) can be assigned to the DIP layer. ${ }^{39}$ Even if the two reflections at $q$ $=2.24 \AA^{-1}$ and $q=2.98 \AA^{-1}$ may be assigned to higherorder reflections from a $d_{l}$ stacking $\left[(006)_{\lambda}\right.$ and $\left.(008)_{\lambda}\right]$, most correspond to lattice spacings $d_{s}<5.5 \AA$ and can be assigned to the UC of the HBS discussed above. However, since we came to the conclusion that the coexisting phases $(\lambda$ and $\sigma$ ) exhibit a fiberlike structure rather than azimuthal order, it is not possible to assign these $\left(d_{s}\right)$ reflections unambiguously to one of the phases. This is indicated by the subscript $\lambda / \sigma$ in Fig. 7 and in Table III, which lists the $\left|q_{\|}\right|$ positions of the reflections discussed above and the derived lattice spacings $d_{\|}$parallel to the surface. The assignment of the reflections to the various crystalline orientations is also indicated in the table. Analyzing the peak widths of these DIP reflections provides a lower limit for the associated domain sizes of $150-250 \AA$ which is of the order of the Augrain size.

By inspection of the intensities of the Bragg reflections associated with the $\lambda$ - and $\sigma$-phases, respectively, we can provide an estimate for the volume ratio of the two phases in the film. For this estimate, the integrated intensity of the strongest $d_{l}$ reflection at $\left|q_{\|}\right|=0.376 \AA^{-1}\left[(001)_{\lambda}, \quad I\right.$ $\left.=0.311 \times 10^{-3}\right]$ is compared to the integrated intensity of the $d_{s}$ reflection at $\left|q_{\|}\right|=1.15 \AA^{-1}\left[(11)_{\lambda / \sigma}, \quad I=0.011\right.$ $\left.\times 10^{-3}\right]$, which has already been observed as $\sigma$-phase inplane reflection in former studies. To obtain an upper limit of the fraction of $\sigma$-phase in the DIP film, we assign the $d_{s}$ peak entirely to the $\sigma$-phase. Assuming that the DIP film is ideally 
TABLE III. Position of the GIXD Bragg reflections of the DIP/Au sample (no. 1). $\left|q_{\|}\right|^{*}$ values in bold letters have already been observed as in-plane reflections of DIP grown in the $\sigma$-phase in previous studies. The calculations have been carried out assuming the following UC parameters: $a=8.55 \AA, b=7.09 \AA$, and $\gamma=90^{\circ}$. Subscripts $\sigma$ and $\lambda$ indicate to which phase the labeled reflections are assigned.

\begin{tabular}{lccc}
\hline \hline $\begin{array}{c}\left|q_{\|}\right|^{*} \\
\left(\AA^{-1}\right)\end{array}$ & $\begin{array}{c}d_{\|} \\
(\AA)\end{array}$ & $\begin{array}{c}\text { Assigned } \\
\text { to }\end{array}$ & $\begin{array}{c}\text { Calculated } \\
\left(\AA^{-1}\right)\end{array}$ \\
\hline $0.375(9)$ & $16.71 \pm 0.05$ & $(001)_{\lambda}$ & \\
$0.753(8)$ & $16.67 \pm 0.05$ & $(002)_{\lambda}$ & \\
$\mathbf{1 . 1 5 3 ( 0 )}$ & $5.45 \pm 0.02$ & $(11)_{\lambda / \sigma}$ & 1.1513 \\
$\mathbf{1 . 4 7 ( 5 )}$ & $4.26 \pm 0.03$ & $(20)_{\lambda / \sigma}$ & 1.4698 \\
$\mathbf{1 . 7 1 ( 9 )}$ & $3.66 \pm 0.03$ & $(21)_{\lambda / \sigma}$ & 1.7163 \\
$1.76(7)$ & $3.56 \pm 0.03$ & $(02)_{\lambda / \sigma}$ & 1.7724 \\
$2.24(1)$ & $16.82 \pm 0.07$ & $(006)_{\lambda}$ & \\
$2.67(4)$ & 2.35 & $\mathrm{Au}(111)$ & \\
$2.980(8)$ & $16.86 \pm 0.07$ & $(008)_{\lambda}$ & \\
$3.08(5)$ & 2.04 & $\mathrm{Au}(200)$ & \\
\hline \hline
\end{tabular}

textured with the long axis perpendicular ( $\sigma$-phase) and parallel ( $\lambda$-phase) to the surface, respectively, but otherwise ideally powderlike (fiber phase), we estimate from the intensity ratio (corrected for polarization and Debye-Waller factors $^{40,41}$ ) that the content of $\sigma$-phase throughout the film is $\$ 26 \%$. This demonstrates that DIP/Au preferentially grows in the $\lambda$-phase.

The observation of a preferred $\lambda$-phase for DIP molecules on $\mathrm{Au}$ in contrast to an almost exclusive $\sigma$-phase growth on $\mathrm{SiO}_{2}$ and $\mathrm{Al}_{2} \mathrm{O}_{3}$ can qualitatively be explained by different substrate-molecule interactions. In the absence of reactive and strongly polar groups on DIP, one can assume that the molecule-molecule, as well as the substrate-molecule, interactions are predominantly of van-der-Waals type. In this picture, the strength of the polarizability of the substrate, which results in image charges of the fluctuating delocalized $\pi$-orbital electrons of the molecules, is crucial for the free energy of the substrate-molecule binding. A large polarizability - as in the present case of a Au substrateresults in a large molecule-substrate binding energy that cannot be compensated by the gain in free energy by molecules standing upright and aligning their $\pi$-orbitals. It must be noted, however, that this picture mainly applies to the growth of the first monolayer. Also, the growth conditions (substrate temperature and deposition rate) may have a significant influence on the molecular orientation in the organic thin film.

Pentacene, another flat aromatic molecule, also forms a standing phase on inert surfaces, ${ }^{42}$ while a metal surface promotes a configuration with the long molecular axis along the surface. ${ }^{43}$ This indicates that the strong dependence of the molecular arrangement (lying or standing phase) on the substrate nature is quite characteristic for these systems.

To complete the discussion of our x-ray scattering data, we note that the reflections at $q=1.22(5) \AA^{-1}$ and at $q$ $=1.40(1)$ in the specular and GIXD scans [see Figs. 6(b) and 7] cannot be explained by the 2D UC proposed so far. We suggest the occurrence of a second coexisting $\lambda^{*}$-phase with a different UC (the best fit is obtained with $a$ $=8.96 \AA, b=6.25 \AA, \gamma=90^{\circ}$; aspect ratio $b / a=1: 1.4336$; area per molecule $=28.0 \AA^{2}$ ). This $\lambda^{*}$-phase does not necessarily exhibit a HBS structure. The coexistence of two $\lambda$-phases is also observed in other organic-on-metal systems, e.g., PTCDA/Ag(111). ${ }^{20,21}$ However, further studies conducted preferably on single-crystalline metal substrates are necessary to confirm the UC parameters of the $\lambda^{*}$-phase

\section{Electronic structure (UPS)}

\section{DIP on gold}

Figure 8(a) shows UPS spectra of sample no. 4 for increasing amounts of DIP (nominal coverages $\Theta$ given on the right-hand side) on a wide energy range (binding energy with respect to the Fermi energy $E_{F}$ of the Au substrate). The work function of the Au film, which served as a substrate for the DIP growth study, was $5.40 \mathrm{eV}$. Its valence electron spectrum is shown as the dashed curve. A new emission feature with binding energy $(\mathrm{BE}) \approx 1.0-1.8 \mathrm{eV}$ appears following the deposition of only $2 \AA$ DIP. Also, the intensity of the gold Fermi edge is reduced. However, little change in the rest of the photoemission spectrum is observed. As $\Theta$ is increased, emission features derived from molecular orbitals of the organic material can be seen, in particular for $\Theta>100 \AA$. Closer inspection shows that photoemission intensity from the substrate (at and below $E_{F}$ ) can still be seen up to $\Theta=200 \AA$. No signal from the substrate can be detected at $300 \AA$.

In order to obtain a more detailed insight into the evolution of the photoemission signal of the organic overlayer without the contribution from the metal substrate, we apply a simple subtraction procedure, assuming that the photoemission spectrum of Au does not change upon DIP deposition. The intensity of UPS spectra for different values of $\Theta$ is then normalized to the emission intensity of the Fermi edge, which remains visible up to $\Theta=200 \AA$. The normalized spectrum of the pristine $\mathrm{Au}$ film is subtracted. The resulting curves are displayed in Fig. 8(b). The dotted line in the figure serves as a guide to the eye for changes in the low BE photoemission onset of the DIP film. The onset and the rest of the spectrum shift gradually by $0.45 \mathrm{eV}$ between $\Theta=2 \AA$ and $\Theta=300 \AA^{44}$ This shift can be attributed to a decrease in screening of the photo-hole when emission occurs further and further from the Au surface. ${ }^{46,47}$ This means that the interface hole-injection barrier from $\mathrm{Au}$ into the first DIP monolayer is $1.0 \mathrm{eV}$. However, holes must overcome the additional $0.45 \mathrm{eV}$ in a "staircase" molecule-to-molecule hopping manner to reach the DIP bulk, and the total holeinjection barrier is $1.45 \mathrm{eV}$.

The total change in the secondary electron cutoff position [top curve of Fig. 8(c)] of $\approx 1.05 \mathrm{eV}$ is established within the first two to three monolayers $(6-12 \AA)$. It corresponds to the amount of the interfacial dipole. In accordance with earlier studies of conjugated organic materials on Au surfaces, the origin of this shift of the vacuum level is attributed to a modification of the Au surface dipole by the adsorption of the organic molecules. ${ }^{48,49}$ Thus, at maximum film thickness $(\Theta=300 \AA)$ the first ionization energy, IE, of DIP is $5.80 \mathrm{eV}$ 

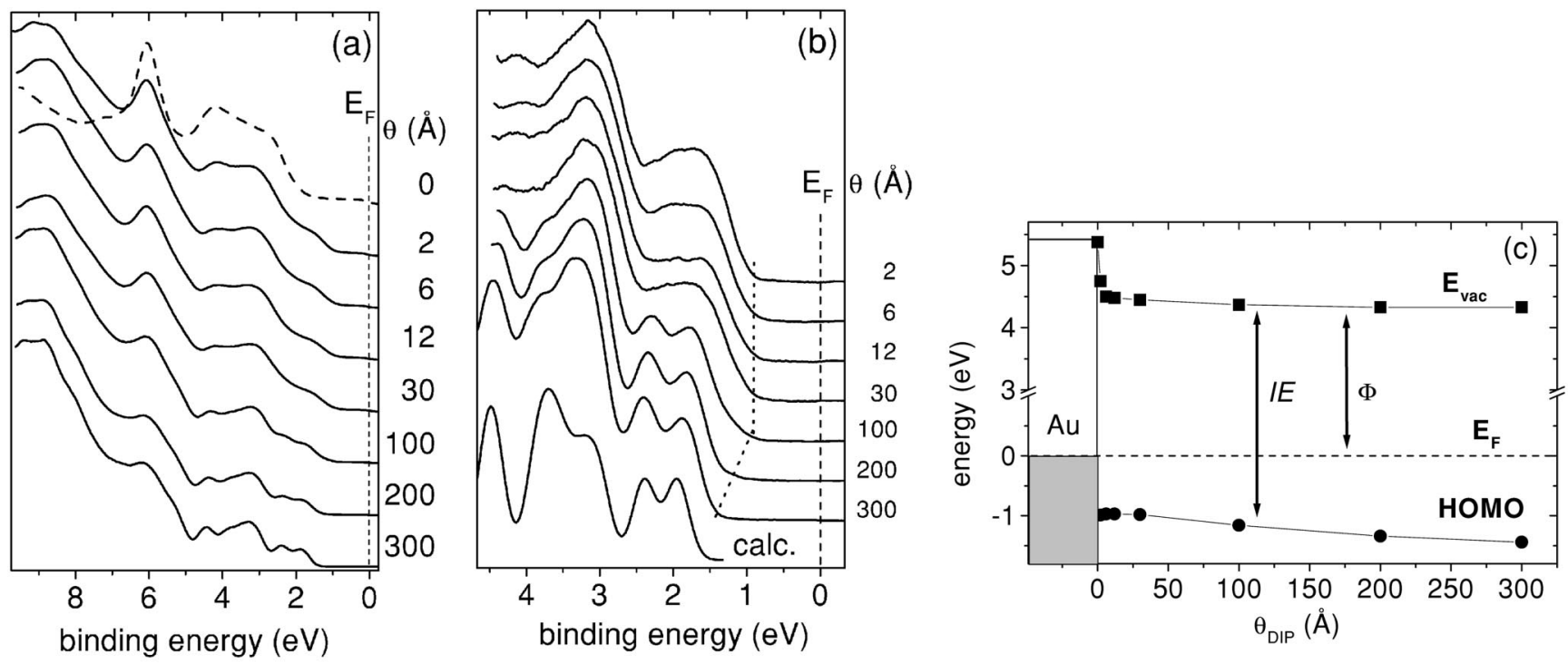

FIG. 8. (a) UPS spectra of sample no. 4 for increasing amounts of DIP $(\Theta)$ on Au in survey scans. (b) UPS spectra of the DIP overlayer on $\mathrm{Au}$, the contribution from the Au substrate has been subtracted. The dotted line is a guide to the eye for the shift of the HOMO-onset. The bottom curve is a calculated UPS spectrum of DIP. (c) Summary of the analysis of the UPS-spectra evolution. The shift of the vacuum level $E_{v a c}$, the shift of the HOMO-position, and the Fermi energy $E_{F}$ are denoted, thus providing an energy-level diagram as a function of nominal DIP coverage $\Theta$. Also, the definition of the sample work function $\Phi$ and the first ionization energy (IE) of the DIP film are shown.

and a change in sample work function $\Phi$ from $5.40 \mathrm{eV}$ (pristine $\mathrm{Au}$ ) to $4.35 \mathrm{eV}$ has been revealed. Figure $8(\mathrm{c})$ summarizes these results and provides an energy-level diagram as a function of $\Theta$ for the DIP/Au interface.

Figure 8(b) shows that little change occurs in the line shape and the onset of the DIP spectra up to $\Theta=12-30 \AA$, and that the HOMO and HOMO-1 of DIP become clearly resolved only for higher coverage. These peaks are centered at $1.85 \mathrm{eV}$ and $2.4 \mathrm{eV} \mathrm{BE}$, respectively, in the $300 \AA$ spectra.

The similarity of shape and HOMO-onset position for the spectra below $\Theta=30 \AA$ points towards completion of the first DIP monolayer at a nominal coverage between $6 \AA$ and 30 $\AA$. This proposition is supported by the rapid decrease of the sample work function in this coverage range [Fig. 8(c)].

The $\Theta=300 \AA$ DIP UPS spectrum is compared to a calculated spectrum obtained from density-functional theory, as described in Sec. II [bottom of Fig. 8(b)]. Given that our simple procedure does not include photoemission cross sections and possible intensity variations induced by molecular orientation, the agreement between calculated and measured spectra is excellent. Furthermore, an evaluation of molecular orbitals shows that the HOMO of DIP is delocalized over the perylene core and the HOMO-1 over the entire long axis of the molecule.

The chemical reactivity of $\mathrm{Au}$ is relatively small and we postulate that DIP physisorbs on the Au surface, similar to most other conjugated organic materials on gold. ${ }^{31,48,50-53}$ However, the UPS spectra at low DIP coverage (up to ca. 30 $\AA$ ) do look somewhat different from the ones obtained from thicker films. For instance, HOMO and HOMO-1 are not resolved. A DIP molecule in direct contact with the Au surface may have a different geometry than one in the DIP crystal bulk, leading to different molecular orbital energies. This change in molecular conformation could also contribute to the total shift of the photoemission onset and change in the spectral shape. We also cannot exclude that poor resolution in the thin-film spectra is merely due to disorder of the molecular film. The polycrystalline Au substrate may provide a number of energetically different adsorption sites for the DIP molecules (e.g., local morphology, crystal surfaces, and grain boundaries). Then for $\Theta<30 \AA$ the UPS spectrum would simply be a superposition of emission from DIP molecules with different adsorption sites.

The fact that emission intensity from the Au film can still be detected up to a nominal DIP coverage of $200 \AA$ is a direct consequence of the pronounced island growth under the present experimental conditions (the elastic mean free path for photoelectrons in our experiment is estimated around 5-10 $\AA$, see Ref. 54). Every UPS spectrum is a superposition of emission from DIP molecules with rather different environments (i.e., different effective film thickness, and thus different photo-hole screening; see above) as well as some emission from Au. This explains the rather broad spectrum observed for $\Theta=100 \AA$ in Fig. 8(b). The low BE photoemission onset is smeared out between $1.0 \mathrm{eV}$ and ca. $1.3 \mathrm{eV}$, hence we detect emission from molecules from the first monolayer (with the strongest screening/and possibly different conformation; $1.0 \mathrm{eV}$ ), and molecules already far from the surface (less efficient screening; $1.3 \mathrm{eV}$ ). As mentioned above, the onset is the sharpest for $\Theta=300 \AA$, indicating that the photoemission signal comes from DIP molecules with more "bulk" properties, i.e., the film consists of closed multilayers. The TEM images of Fig. 4 support the idea that at maximum $\Theta$ sample areas with very low effective DIP coverage are unlikely.

\section{Gold on DIP}

Having established that no charging occurs for the $300 \AA$ thick DIP film, ${ }^{44}$ the study of Au on DIP was conducted. 

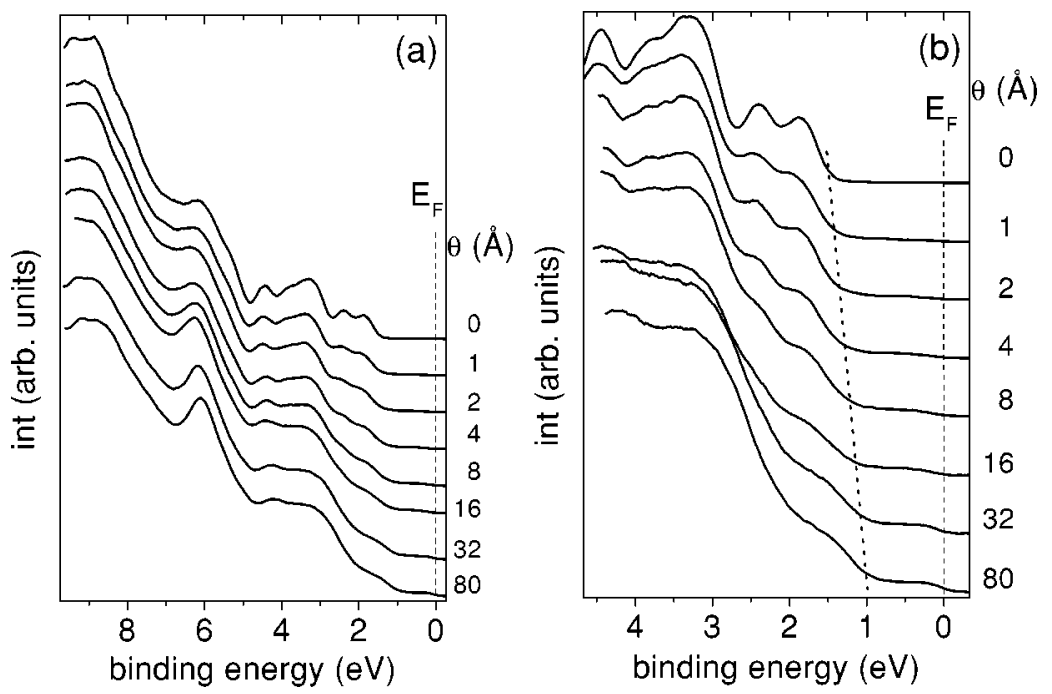

FIG. 9. UPS spectra for increasing amounts of $\mathrm{Au}(\Theta)$ on DIP in a survey (a) and a close-up on the low binding-energy region (b). The dotted line in (b) is a guide to the eye for the shift of the HOMO onset. The spectra (except $\Theta=0$ and 80 $\AA$ ) were recorded during illumination with visible light at saturation conditions (see text).

Because of sample charging during UPS at low Au coverage, a discharging technique, described in detail in Ref. 32, must be applied. In brief, small Au clusters on top of the DIP film exhibit positive charging, since electron transfer from surrounding DIP to the clusters, in order to establish charge neutrality, is difficult. Optical excitation of DIP by simultaneous irradiation of the sample with light leads to the desired electron transfer from the excited organic molecules to the charged Au clusters, and allows for complete discharging.

The evolution of the valence electron spectrum of DIP upon increasing amounts of Au deposition is shown in Fig. 9(a) as survey and (b) as close-up on the low BE region. Spectra with $\mathrm{Au}$ coverage $\Theta$ between $1 \AA$ and $32 \AA$ were recorded, while light from a standard halogen lamp was focused onto the sample. The light intensity was optimized in order to completely compensate the charging mentioned above. ${ }^{32}$ Overall, the sequence of spectra looks almost like a reversal of the study of DIP on Au, and we propose that the interaction between DIP and the deposited $\mathrm{Au}$ is of physisorptive nature. ${ }^{48,50,52,53}$ However, we could not apply a subtraction procedure analogous to the one described above [see Fig. 8(b)], since the size distribution of the Au clusters, which critically determines their electronic structure, is unknown. As more Au is deposited, the spectral features of DIP become less prominent, and a finite density of valence states (DOVS) is observed within the energy gap of the organic material. However, this DOVS does not extend up to $E_{F}$ until the $\mathrm{Au}$ coverage reaches $80 \AA$, where a true metallic Fermi edge is observed. Starting from ca. $\Theta=16 \AA$, a small tail-like DOVS at $E_{F}$ is detected. We attribute this to the average cluster size distribution that is present at individual deposition steps. Whereas most clusters at and below $\Theta=16$ $\AA$ are too small to be metallic, an increasing number of the clusters become large enough to exhibit metallic character. $^{55,56}$ This behavior is comparable to that observed for other physisorptive metal/organic systems. ${ }^{35,57-59}$ At $\Theta=80$ $\AA$ most of the Au deposit has become metallic and the spectrum closely resembles the one obtained for $2 \AA$ DIP on Au. A clear Fermi edge is visible, and the prominent photoemission peak at $6 \mathrm{eV} \mathrm{BE}$ is attributed to the Au $5 d$ band. Note that the low BE onset from the DIP HOMO exhibits the reverse gradual trend observed for DIP on Au, i.e., shifting from $1.45 \mathrm{eV}(0 \AA \mathrm{Au})$ to $1.0 \mathrm{eV}(80 \AA \mathrm{Au})$. This is a clear demonstration that screening of the molecule photo-hole by the metal is responsible for the observed shift. By increasing the average Au cluster size, we gradually "switch on" metallicity, i.e., increase the polarizability, of the matter next to DIP molecules. ${ }^{32,60-62}$ Thus, the electronic properties and energy-level alignment of interfaces formed by deposition of DIP onto gold, and those formed by deposition of $\mathrm{Au}$ onto DIP are identical, despite different interface morphologies.

We did not continue to deposit more Au on the sample to arrive at a spectrum of Au only, since the growth study appears to be complete at this point. Any further deposition of $\mathrm{Au}$ would simply result in a spectrum like the one shown in Fig. 8(a), and no new information about the interface could be obtained.

\section{CONCLUSION}

We have investigated morphology, crystalline structure, and the electronic structure of interfaces formed between $\mathrm{Au}$ and DIP, using AFM, cross-sectional TEM, x-ray diffraction, and UPS. With AFM we show that DIP/Au exhibits pronounced islanding morphology under the present growth conditions. Cross-sectional TEM of Au/DIP/Au heterostructures confirms the islanding and demonstrates that the $\mathrm{Au}$ layer on top of the organic film is formed by coalescence of $\mathrm{Au}$ clusters rather than by a layer-by-layer growth. Some Au clusters diffusion into the DIP islands can also be observed. Remarkably, individual monolayers of DIP can be discerned in some DIP islands in the TEM images, pointing to high structural order of DIP molecules exhibiting $\lambda$-phase growth. This observation is in contrast to $\mathrm{DIP} / \mathrm{SiO}_{2}$ where the molecules grow (almost exclusively) in the $\sigma$-phase. Furthermore, x-ray-diffraction measurements on DIP/Au samples parallel and perpendicular to the surface provided evidence for the coexistence of $\lambda$-phase and $\sigma$-phase. A quantitative estimate indicates preferential $\lambda$-phase growth $(\gtrsim 74 \%)$. Compared to $\mathrm{DIP} / \mathrm{SiO}_{2}$ and $\mathrm{DIP} / \mathrm{Al}_{2} \mathrm{O}_{3}$, the observation of pronounced islanding and preferential molecular orientation in the $\lambda$-phase can be explained by different van-der-Waals 
substrate-molecule interactions, i.e., insulating vs metal substrates.

The evolution of the UPS spectra with increasing amount of DIP is strongly influenced by islanding. The first DIP monolayer on $\mathrm{Au}$ forms rather instantaneously $(\Theta \leqslant 30 \AA)$ but the spectra exhibit "bulk" properties only for nominal coverages $\Theta>200 \AA$. Furthermore, the photoemission data reveal that DIP physisorbs on $\mathrm{Au}$, and a total hole-injection barrier of $1.45 \mathrm{eV}$ is measured. For Au deposition onto DIP we find evidence for the formation of Au clusters as already observed by TEM, which percolate only for Au coverages greater than $32 \AA$, to give comprehensive metal surface conductivity. The nature of the interaction between the Au clusters and DIP is also found to be physisorptive, and the interface energy-level alignment is independent of the deposition sequence, despite the very different interface morphologies.

\section{ACKNOWLEDGMENTS}

The UPS-synchrotron work was supported by the IHPContract No. HPRI-CT-1999-00040 of the European Com- mission. Part of this work was supported by a grant of the NSF (Grant No. DMR-0097133), by the New Jersey Center for Organic Optoelectronics, and by the MRSEC program of the NSF (Grant No. DMR-98-09483). We acknowledge financial support of the Interuniversity Research Projects (PAI/ IUA) on "Sciences of Interfacial and Mesoscopic Structures" sponsored by the Belgian Prime Minister's Office (Federal Services for Scientific, Technical and Cultural Affairs). R.L.J. acknowledges financial support from the Bundesministerium für Bildung, Wissenschaft, Forschung und Technologie (BMBF) Project No. 05 KS1GUC/3. J.G. acknowledges support by the NFSR (Belgium). F.S. acknowledges support within the DFG focus program "Organische Feldeffekt-Transistoren." We thank Robert A. Pascal, Jr. for help with the calculations, U. Täffner for help with the AFM-measurements, N. Karl for providing purified DIP molecules and helpful discussions, U. Habermaier and B. Lemke for fabrication of the Au substrates, and B. Nickel for fruitful discussions and critical reading of the manuscript. A. Stierle and R. Weigel provided excellent support at the MPIbeamline at ANKA.
*Corresponding author. FAX: +49 (0) 711689 1902; Electronic address: Arndt.Duerr@mf.mpg.de; URL: http://dxray.mpistuttgart.mpg.de/site/index_en.html

†Present address: Institut für Physik, Humboldt-Universität Berlin, Newtonstrasse 15, 12489 Berlin, Germany.

${ }^{\ddagger}$ Present address: Physical and Theoretical Chemistry Laboratory, University of Oxford, South Parks Road, Oxford OX1 3QZ, United Kingdom.

${ }^{1}$ C.W. Tang and S.A. v. Slyke, Appl. Phys. Lett. 51, 913 (1987).

${ }^{2}$ Handbook of Conducting Polymers, 2nd ed., edited by $\mathrm{T}$. Skotheim, R. Elsenbaumer, and J. Reynolds (Marcel Dekker, New York, 1997).

${ }^{3}$ R.H. Friend, R.W. Gymer, A.B. Holmes, J.H. Burroughes, R.N. Marks, C. Taliani, D.D.C. Bradley, D.A. Dos Santos, J.L. Bredas, M. Logdlund, and W.R. Salaneck, Nature (London) 397, 121 (1999).

${ }^{4}$ S. Forrest, P. Burrows, and M. Thompson, IEEE Spectrum 37, 29 (2000).

${ }^{5}$ G.H. Gelinck, T.C.T. Geuns, and D.M. de Leeuw, Appl. Phys. Lett. 77, 1487 (2000).

${ }^{6}$ C.D. Dimitrakopoulos and P.R.L. Malenfant, Adv. Mater. (Weinheim, Ger.) 14, 99 (2002).

${ }^{7}$ Y. Gao, K.T. Park, and B.R. Hsieh, J. Appl. Phys. 73, 7894 (1993).

${ }^{8}$ P. Dannetun, M. Fahlman, C. Fauquet, K. Kaerijama, Y. Sonoda, R. Lazzaroni, J.L. Bredas, and W.R. Salaneck, Synth. Met. 67, 133 (1994).

${ }^{9}$ G. Greczynski, M. Fahlman, and W.R. Salaneck, J. Chem. Phys. 113, 2407 (2000).

${ }^{10}$ C. Shen, A. Kahn, and J. Schwartz, J. Appl. Phys. 89, 449 (2001).

${ }^{11}$ M.G. Mason, C.W. Tang, L.S. Hung, P. Raychaudhuri, J. Madathil, D.J. Giesen, L. Yan, Q.T. Le, Y. Gao, S.T. Lee, L.S. Liao, L.F. Cheng, W.R. Salaneck, D.A. dos Santos, and J.L. Bredas, J. Appl. Phys. 89, 2756 (2001).

${ }^{12}$ Conjugated Polymer and Molecular Interfaces: Science and Technology for Photonic and Optoelectronic Applications, ed- ited by W. R. Salaneck, K. Seki, A. Kahn, and J.-J. Pireaux (Marcel Dekker, New York, 2001).

${ }^{13}$ A.C. Dürr, F. Schreiber, M. Kelsch, H.D. Carstanjen, and H. Dosch, Adv. Mater. (Weinheim, Ger.) 14, 961 (2002).

${ }^{14}$ A.C. Dürr, F. Schreiber, M. Münch, N. Karl, B. Krause, V. Kruppa, and H. Dosch, Appl. Phys. Lett. 81, 2276 (2002).

${ }^{15}$ N. Karl, K.-H. Kraft, J. Marktanner, M. Münch, F. Schatz, R. Stehle, and H.-M. Uhde, J. Vac. Sci. Technol. A 17, 2318 (1999).

${ }^{16}$ N. Karl, in Charge Carrier Mobility in Organic Crystals, Organic Electronic Materials Vol. II, edited by R. Farchioni and G. Grosso (Springer, Berlin, 2001), p. 283.

${ }^{17}$ A.C. Dürr, F. Schreiber, M. Kelsch, H.D. Carstanjen, H. Dosch, and O.H. Seeck, J. Appl. Phys. 93, 5201 (2003).

${ }^{18}$ C.B. France, P.G. Schroeder, and B.A. Parkinson, Nano Lett. 2, 693 (2002).

${ }^{19}$ T. Schmitz-Hübsch, T. Fritz, F. Sellam, R. Staub, and K. Leo, Phys. Rev. B 55, 7972 (1997).

${ }^{20}$ B. Krause, A.C. Dürr, K.A. Ritley, F. Schreiber, H. Dosch, and D. Smilgies, Appl. Surf. Sci. 175-176, 332 (2001).

${ }^{21}$ B. Krause, A.C. Dürr, K. Ritley, F. Schreiber, H. Dosch, and D. Smilgies, Phys. Rev. B 66, 235404 (2002).

${ }^{22}$ K. Seki, N. Hayashi, H. Oji, E. Ito, Y. Ouchi, and H. Ishii, Thin Solid Films 393, 298 (2001).

${ }^{23}$ R. Feidenhans'l, Surf. Sci. Rep. 10, 105 (1989).

${ }^{24} \mathrm{H}$. Dosch, Critical Phenomena at Surfaces and Interfaces (Springer, Berlin, 1992).

${ }^{25}$ M. Tolan, X-Ray Scattering from Soft-Matter Thin Films (Springer, Berlin, 1999).

${ }^{26}$ We use polycrystalline $\mathrm{Au}$ to be as close as possible to the "realworld" situation (i.e., for the application in devices), where single crystal Au would probably not be used.

${ }^{27}$ The roughness of the so prepared substrates is $\leqslant 10 \AA$ as determined by AFM and by x-ray reflectivity measurements.

${ }^{28}$ A. C. Dürr, F. Schreiber, M. Kelsch, and H. Dosch, Ultramicroscopy (to be published).

${ }^{29}$ A. Stierle and H. Dosch in ANKA Beamline Book, edited by J. 
Göttlicher and M. Hagelstein (Forschungszentrum Karlsruhe, Karlsruhe, 2002), see p. 34; http://hikwww1.fzk.de/iss/ beamlinebook.html

${ }^{30}$ R.L. Johnson and J. Reichardt, Nucl. Instrum. Methods Phys. Res. 208, 719 (1983).

${ }^{31}$ I.G. Hill, A. Rajagopal, and A. Kahn, J. Appl. Phys. 84, 3236 (1998).

${ }^{32}$ N. Koch, A.C. Dürr, J. Ghijsen, R.L. Johnson, J.-J. Pireaux, J. Schwartz, F. Schreiber, H. Dosch, and A. Kahn, Thin Solid Films 441, 145 (2003).

${ }^{33}$ M. J. Frisch et al., computer code GAUSSIAN, Gaussian Inc., Pittsburgh, PA, 1998.

${ }^{34}$ J. B. Foresman and A. Frisch, Exploring Chemistry with Electronic Structure Methods, 2nd ed. (Gaussian Inc., Pittsburgh, PA, 1996), p. 19.

${ }^{35}$ N. Koch, L.M. Yu, V. Parente, R. Lazzaroni, R.L. Johnson, G. Leising, J.J. Pireaux, and J.L. Bredas, Adv. Mater. (Weinheim, Ger.) 10, 1038 (1998).

${ }^{36}$ A.C. Dür, F. Schreiber, K.A. Ritley, V. Kruppa, J. Krug, H. Dosch, and B. Struth, Phys. Rev. Lett. 90, 016104 (2003).

${ }^{37}$ G.R. Desiraju and A. Gavezzotii, Acta Crystallogr., Sect. B: Struct. Sci. B45, 473 (1989).

${ }^{38}$ A. C. Dürr, Ph.D. thesis, Universität Stuttgart, 2002, http:// elib.uni-stuttgart.de/opus/volltexte/2002/1215/

${ }^{39}$ This has been checked by scans of $\alpha_{i}$ at the position of individual Bragg reflections which exhibit a maximum at $\alpha_{i}=\alpha_{c \text {,DIP }}$ $=0.15^{\circ}$ if associated to DIP. For $\left|q_{\|}\right|<2.67 \AA^{-1}$ no Au reflections are allowed. A detector scan for $\left|q_{\|}\right| \gtrsim 2.60 \AA^{-1}$ at $\alpha_{i}$ $=0.7^{\circ}$, which enhances the intensity contribution of the substrate by approximately one order of magnitude, allows one to clearly identify the Au substrate reflections.

${ }^{40}$ A. Rühm, Ph.D. thesis, Universität Wuppertal, 1998.

${ }^{41}$ In GIXD geometry a correction factor of $1 / \tan ^{2}(2 \Theta)$ has to be applied, which includes the polarization correction. Since we do not know the thermal mean-square displacement $\sqrt{\left\langle u^{2}\right\rangle}$ in the Debye-Waller factor (DWF) $\left[\exp \left(-q^{2}\left\langle u^{2}\right\rangle\right]\right.$ for DIP in the different crystallographic orientations we assume that $\sqrt{\left\langle u^{2}\right\rangle}$ is independent of the crystallographic direction; in this case the DWF has actually no influence on the intensity ratio.

${ }^{42}$ R. Ruiz, B. Nickel, N. Koch, L.C. Feldman, R.F. Haglund, A. Kahn, and G. Scoles, Phys. Rev. B 67, 125406 (2003).

${ }^{43}$ L. Casalis, M.F. Danisman, B. Nickel, G. Bracco, T. Toccoli, S. Iannotta, and G. Scoles, Phys. Rev. Lett. 90, 206101 (2003).

${ }^{44}$ There is an apparent rigid shift of $0.05 \mathrm{eV}$ of the UPS spectra towards higher $\mathrm{BE}$ when going from $\Theta=200 \AA$ to $\Theta=300 \AA$.
One might speculate that this shift is due to charging of the organic surface. However, a curve fitting analysis of these two spectra reveals that the width of the HOMO and HOMO-1 peaks is actually smaller for the thicker film. This is in contrast to the expected broadening of photoemission spectra when sample charging is present (Ref. 45), thus we conclude that charging is not occurring.

${ }^{45}$ N. Koch, D. Pop, R.L. Weber, N. Böwering, B. Winter, M. Wick, G. Leising, I.V. Hertel, and W. Braun, Thin Solid Films 391, 81 (2001).

${ }^{46}$ E.V. Tsiper and Z.G. Soos, Phys. Rev. B 64, 195124 (2001).

${ }^{47}$ E.V. Tsiper, W. Gao, Z.G. Soos, and A. Kahn, Chem. Phys. Lett. 360, 47 (2002).

${ }^{48}$ H. Ishii, K. Sugiyama, E. Ito, and K. Seki, Adv. Mater. (Weinheim, Ger.) 11, 605 (1999).

${ }^{49}$ N. Koch, J. Ghijsen, A. Elschner, R.L. Johnson, J.-J. Pireaux, J. Schwartz, and A. Kahn, Appl. Phys. Lett. 82, 70 (2003).

${ }^{50}$ H. Oji, E. Ito, M. Furuta, K. Kajikawa, H. Ishii, Y. Ouchi, and K. Seki, J. Electron Spectrosc. Relat. Phenom. 101-103, 517 (1999).

${ }^{51}$ H. Proehl, M. Toerker, F. Sellam, T. Fritz, K. Leo, C. Simpson, and K. Müllen, Phys. Rev. B 63, 205409 (2001).

${ }^{52}$ N.J. Watkins, L. Yan, and Y. Gao, Appl. Phys. Lett. 80, 4384 (2002).

${ }^{53}$ H. Peisert, M. Knupfer, T. Schwieger, J.M. Auerhammer, M.S. Golden, and J. Fink, J. Appl. Phys. 91, 4872 (2002).

${ }^{54}$ C.D. Wagner, W.M. Riggs, L.E. Davis, J.F. Moulder, and G.E. Muilenberg, Handbook of X-ray Photoelectron Spectroscopy (Perkin-Elmer, Eden Prairie, 1978).

${ }^{55}$ C.N.R. Rao, V. Vijayakrishnan, H.N. Aiyer, G.U. Kulkarni, and G.N. Subbanna, J. Phys. Chem. 97, 11157 (1993).

${ }^{56}$ C. Xu, W.S. Oh, G. Liu, D.Y. Kim, and D.W. Goodman, J. Vac. Sci. Technol. A 15, 1261 (1997).

${ }^{57}$ N. Koch, R. Pairleitner, Q.T. Le, E.W. Forsythe, Y. Gao, and G. Leising, Appl. Phys. Lett. 76, 3738 (2000).

${ }^{58}$ N. Koch, E. Zojer, A. Rajagopal, J. Ghijsen, R.L. Johnson, G. Leising, and J.J. Pireaux, Adv. Funct. Mater. 11, 51 (2001).

${ }^{59}$ N. Koch, J. Ghijsen, R.L. Johnson, J. Schwartz, J.-J. Pireaux, and A. Kahn, J. Phys. Chem. B 106, 4192 (2002).

${ }^{60}$ O.D. Haberlen, S.C. Chung, and N. Rosch, Ber. Bunsenges. Phys. Chem. 98, 882 (1994).

${ }^{61}$ T.G. Schaaff, M.N. Shafigullin, J.T. Khoury, I. Vezmar, R.L. Whetten, W.G. Cullen, P.N. First, C. GutierrezWing, J. Ascensio, and M.J. JoseYacaman, J. Phys. Chem. B 101, 7885 (1997).

${ }^{62}$ M.A. Omary, M.A. Rawashdeh-Omary, C.C. Chusuei, J.P. Fackler, and P.S. Bagus, J. Chem. Phys. 114, 10695 (2001). 